\title{
Mucinous adenocarcinoma of the prostate: case report and review of the literature
}

\begin{abstract}
Background: Mucinous adenocarcinoma of the prostate (MACP) is a rare variant of prostate carcinoma that is characterized by pools of extra-acinar mucin. Precise diagnosis is important due to nonspecific findings on imaging tests, its aggressive behaviour and poor response to radiotherapy. The essential point in the diagnosis of MACP is to rule out the extraprostatic malignancies, originating from the bladder or colorectum.
\end{abstract}

Case report: A 57-year-old man presented to our clinic with urinary frequency, nocturia and voiding difficulties. Digital rectal examination revealed a slightly enlarged prostate, without palpable nodules. Patient PSA was $18.0 \mathrm{ng} / \mathrm{mL}$. Twelvecore transrectal ultrasound-guided biopsy confirmed prostate cancer with Gleason score $8(3+4)$. Up to $50 \%$ of the tumor lesion consisted of neoplastic glands with isolated cells, floating in mucinous material. The metastatic work-up, including CT scan and bone scintigraphy was negative and radical retropubic prostatectomy with lymph node dissection has been performed. Periodic Acid Schiff staining confirmed the presence of mucinous prostatic adenocarcinoma. Morphological examination was negative for lymph nodes metastases. The extraprostatic extension and surgical margins were negative. Three years after surgery, patient's serum PSA remained undetectable, without recurrence.

Conclusion: We report this case due to the rarity of primary MACP and its challenging diagnosis. Although MACP may be associated with poor outcome, its proper diagnosis and treatment significantly contribute to favorable prognosis and patient survival.
Volume 6 Issue 2 - 2018

\author{
Alexander Otsetov,' Kalin Kalchev, ${ }^{2}$ Natasha \\ Takova, ${ }^{3}$ Alexander Hinev ${ }^{4}$ \\ 'Department of Pathology, Umeå University, Sweden \\ ${ }^{2}$ Department of Pathology, Varna Medical University, Bulgaria \\ ${ }^{3}$ Department of Urology, Centre Hospitalier Saint-Dié des \\ Vosges, France \\ ${ }^{4}$ Department of Urology, Varna Medical University, Bulgaria
}

Correspondence: Alexander Otsetov, Department of Pathology, Institute for Medical Biosciences,Umeå University, Umeå, Sweden, Tel +46 70 234-2829, Email aleksandar.otsetov@umu.se

Received: February 20, 2018 | Published: March 12, 2018

Keywords: prostate cancer, mucinous adenocarcinoma, mucin

Abbreviations: MACP, mucinous adenocarcinoma; PSA, prostate specific antigen; CT, computed tomography; TNM; NM, classification of malignant tumours; MRI, magnetic resonance imaging

\section{Introduction}

Mucinous adenocarcinomas of the prostate (MACP) are rare malignant tumors, characterized by the pools of extraluminal mucin, accounting less than $1 \%$ of all types of prostate cancer $(\mathrm{PCa}) .{ }^{1}$ Because 60 to $90 \%$ of $\mathrm{PCa}$ also secrete mucus, the diagnosis of primary MACP relies on the previously reported histologic criteria. ${ }^{2}$ The defining criteria for this tumor were based on the presence $>25 \%$ of mucinous component of the tumor. ${ }^{3,4}$ MACP is associated with increased levels of prostate-specific antigen (PSA), metastatic spread to bones and responds to hormonal therapy. ${ }^{3} \mathrm{MACP}$ originating from the urinary bladder, urethra, or colon, invade the prostate and can mimic MACP. The infiltrating component in these cancers also contains lakes of mucin. ${ }^{4,5}$ Epstein et al. ${ }^{6}$ divided MCAP into three groups:

i. Mucinous adenocarcinoma

ii. Primary signet-ring cell carcinoma

iii. Mucinous carcinoma with signet-ring cells. ${ }^{3} \mathrm{MCAP}$ are associated with elevated PSA levels, well responding to hormonal therapy. In contrast, the signet-ring cell and mucinous carcinomas with signet ring cells do not respond to hormonal therapy. In addition, it was found that primary signet ring cell carcinoma and mucinous carcinoma with signet ring cells had poor outcome, compared with mucinous adenocarcinoma without signet ring cells. ${ }^{6}$

\section{Case presentation}

A 57-year-old man without history for malignant diseases, presented to our hospital with urinary frequency, nocturia and difficulties to void. Digital rectal examination revealed a slightly enlarged prostate (around $40 \mathrm{cc}$ ) without palpable nodules. The serum PSA was $18.0 \mathrm{ng} /$ $\mathrm{mL}$. Twelve-core transrectal ultrasound-guided biopsy confirmed PCa with Gleason score $8(3+4)$. Seven of the 12 cores contained acinar adenocarcinoma, occupying up to $25 \%$ of the prostatic tissue, with the pattern 4 dominant. In addition, $50 \%$ of the neoplastic glands contained cells, floating in a mucinous material. The metastatic work-up (CT scan and bone scintigraphy) was negative. Open radical prostatectomy with bilateral pelvic lymph node dissection was performed. The intraoperative frozen section of the lymph nodes and surgical margins were negative for metastatic disease. Radical prostatectomy specimens and the lymph nodes have been examined in a standard fashion. The prostatectomy specimen weighted $45 \mathrm{~g}$. Prostatic tissue was largely nodular with yellow areas, involving the posterior zone. Microscopically the neoplastic proliferation was found in $40 \%$ of the prostate. Gleason was scored as $8 / 10(3+4)$. In addition, pattern 5 was found in $2 \%$ of prostatic tissue. All pelvic lymph nodes were negative for metastases. The extraprostatic extension and surgical margins were also negative. The histological examination showed mostly 
acinar prostatic adenocarcinoma, consisting $40 \%$ of neoplastic glands and single cells, floating in abundant mucinous material, confirmed by a positive periodic Acid Schiff(PAS) staining. The rest of the prostatic specimen showed benign prostatic hyperplasia (BPH) with high-grade intraepithelial neoplasia (HGPIN). The final diagnosis was mucinous adenocarcinoma, pT2cN0M0 and Gleason score 8/10 (3+4), as shown in Figure 1.

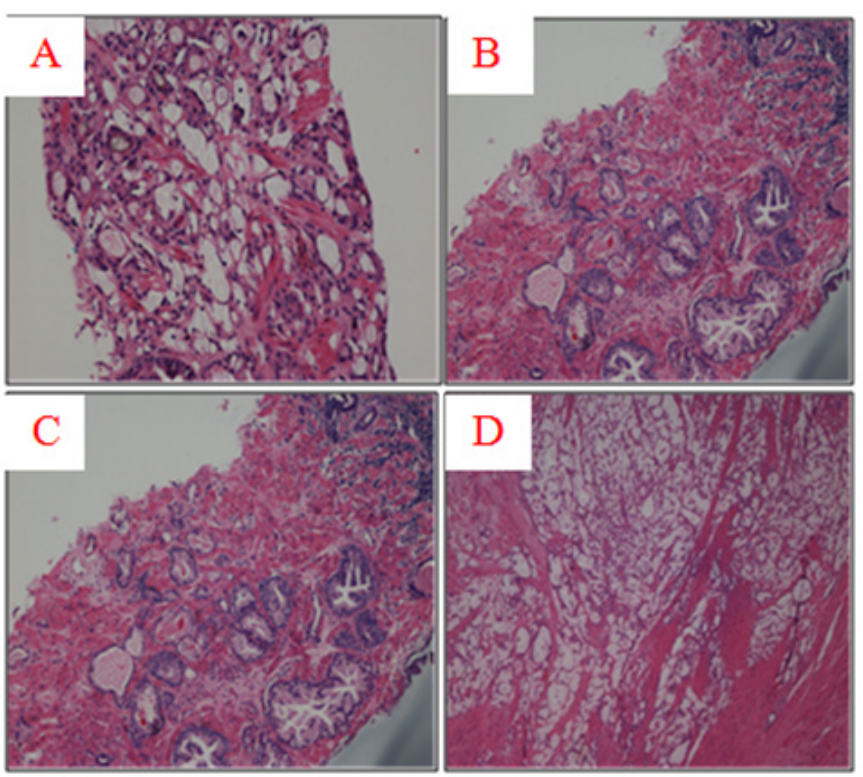

Figure 1 (A) Tru-cut biopsy specimen with abundant extracellular mucin more than $25 \%$ that defines the tumor as mucinous (H \& E, x100). (B) The same tru-cut biopsy with evidence of BPH and HGPIN (H \& E, x 100). (C) Higher magnification shows mucionous adenocarcinoma with glandular fusion, Gleason score $8(3+4)$. Pools of mucin with single cells floating in them are clearly visible (H \& E, x200). (D) Radical prostatectomy specimen with vast areas of mucinous adenocarcinoma (H \& E, x40).

Due to specific pathology, we performed extensive metastatic work-up, including colonoscopy, which was negative. In addition, the stool test for occult bleeding and CEA antigen were in normal range. Three years after surgery and close follow-up, patient has normal serum PSA levels with acid phosphatase within normal range.

\section{Discussion}

Samaratunga et al. ${ }^{9}$ have described MACP in 1882 and since then, less than 200 cases have been reported. ${ }^{1}$ The diagnosis of MACP relies on extraluminal pools of mucin found in more than $25 \%$ in prostatic tissue. $^{2}$ The mean age at the time of diagnosis is usually less than 60 years old. In overall the pre-treatment PSA levels are between 4.0 and $10.0 \mathrm{ng} / \mathrm{mL}$ and TNM is T1c, T2a or T2b. ${ }^{3-5}$ Grading of MACP is controversial and some authors suggested scoring the tumor based on the underlying architecture pattern, ignoring the extracellular mucin. Current recommendations for grading mucinous cancers are to grade the underlying architecture, based on extravasated mucin, essential criteria for the diagnosis of mucinous adenocarcinoma. ${ }^{7}$ Recently, an updated grading of MACP has been proposed, based on the underlying architectural pattern, i.e. well-formed glands or Gleason 3; cribriform glands, or Gleason 4; single cells or Gleason 5, etc. ${ }^{8}$ Most commonly, MACP are Gleason $8(4+4){ }^{4,9}$ In a recent study of 143 cases with mucinous adenocarcinomas, the mean age was $61.4 \mathrm{y} / \mathrm{o}$, and the mean preoperative PSA $7.8 \mathrm{ng} / \mathrm{ml}$. Tumors in stage $\mathrm{cT} 1$ were $81 \%$, compared with these in cT2 (19\%). The vast majority of mucin consisting carcinomas were with Gleason $4+3$ (54.5\%). In another study with 73 cases, mucinous component was found in more than $25 \%{ }^{9}$ Morphologically, MACP demonstrate mucoid or gelatinous cut surface and the light microscopy reveals pools of mucin in the stroma with groups of cells, forming acini. The presence of luminal mucin is a specific feature of these tumors. Lately, important data have been provided about the characteristics and distribution of mucin in both normal and malignant prostatic tissues. ${ }^{10,11}$ The immunostaining in benign tissues is positive for neutral mucins, whereas carcinomas contain sulphated type of sialic acidic mucin. The benign normal prostate does not secrete acidic mucin, which is a feature of most $\mathrm{PCa}$, which however, is secreted in a lesser extent. Colloid cancers also produce this type of mucin but in a greater extent. ${ }^{10}$ Another study reported that the mucin in MACP is much more than the luminal mucin in the acinar carcinoma Gleason 3, demonstrating difference between both prostatic carcinomas. ${ }^{7}$ Single cells, including signet ring forms, neuroendocrine and Paneth-like cells are also frequent findings in MACP. Immunohystochemically, MACP are positive for prostate specific antigen (PSA), prostatic acid phosphatise (PAP) and low molecular weight cytokeratins (LMWCK). Pure MACP are negative for carcinoembryonic antigen (CEA) and high molecular weight cytokeratins (HMWCK). ${ }^{7}$ Compared with MACP, the urothelial carcinoma is positive to HMVCK and CK $7 / 20$ and negative for PSA and PAP. ${ }^{5}$ However, the imaging diagnostic tools, like CT-scan and MRI, which otherwise are currently used in the clinical practice, for the imaging of MACPs have been suggested as non-specific, because the visualization of mucinous adenocarcinoma is difficult on MRI. ${ }^{12-15}$

MACP outcome and prognostic significance are disputable and not fully understood. ${ }^{13}$ Previously, Epstein and al have reported six cases with aggressive biological behaviour with propensity to develop bone metastases. ${ }^{3}$ In another study of Ro et al. ${ }^{17} 12$ cases with high-stage mucinous adenocarcinoma were treated with radiation, hormonal therapy or in a combination, bone metastases were also common. ${ }^{16}$ In contrast, Osunkoya et al. ${ }^{20}$ have reported 5 -year progression free risk of $97.2 \%$ of cases in a group of 47 patients with mucinous prostatic adenocarcinoma. ${ }^{6,8,10}$ Importantly, the diagnosis of MACP obligates to rule out mucinous carcinoma, originating from the gastro-intestinal system and detailed medical history, endoscopy and imaging are important for the patient outcome. Genetic abnormalities also have been detected in MACPs. For example, studies have shown that ERG gene expression occurs in $50 \%$ of mucinous adenocarcinoma. ${ }^{9,17}$ Likewise, TMPRSS2: ERG fusion gene was identified in $83 \%$ of these prostate cancers. ${ }^{18,19}$ Furthermore, MUC 2 immunoexpression was found in the mucinous elements of MACP. ${ }^{20} \mathrm{New}$ study of Osunkoaya et al. ${ }^{20}$ showed that MACPs also can express PTEN in most cases. ${ }^{8}$ Interestingly, the expression of PTEN in MACP was suggested as plausible factor that possibly might explains the attenuated aggressiveness of these tumors, compared with conventional adenocarcinomas of the prostate without extraluminal component. ${ }^{8}$

\section{Conclusion}

Here we present a rare case of mucinous adenocarcinoma and we outline the most important diagnostic features of MACP with specific aspects in the primary diagnosis of this tumor, which every urologist should keep in mind. 


\section{Acknowledgements}

We dedicate this paper in memoriam to our colleague, friend and teacher Prof. Alexander Hinev, suddenly deceased in November 2017.

\section{Conflict of interest}

There is none of the conflict.

\section{References}

1. Fletcher CDM. Diagnostic histopathology of tumors. $4^{\text {th }}$ ed. ChurchillLivingstone Elsevier; 2008. p. 778-779.

2. Elbadawi A, Craig W, Linke CA, et al. Prostatic mucinous carcinoma. Urology. 1979;(13):658-666.

3. Epstein JI, Lieberman PH. Mucinous adenocarcinoma of the prostate gland. Am J Surg Pathol. 1985;9(4):299-308.

4. Humphrey P, Amin MB, Berney D, et al. Pathology and genetics: tumors of the urinary system and male genital organs. WHO classification of tumors. $4^{\text {th }}$ ed. Zurich, Switzerland: IARC Press; 2016:136-150.

5. Grignon DJ. Unusual subtypes of prostate cancer. Mod Pathol. 2004;17(3):316-327.

6. Epstein JI, Allsbrook WC, Amin MB, et al. The ISUP Grading Committee. The 2005 International Society of Urological Pathology (ISUP) consensus conference on Gleason grading of prostatic carcinoma. Am J Surg Pathol. 2005;29(9):1228-1242.

7. Epstein JI, Egevad L, Amin MB, et al. the ISUP Grading Committee The 2014 International Society of Urological Pathology (ISUP) consensus conference on Gleason grading of prostatic carcinoma: definition of grading patterns and proposal for a new grading system. Am J Surg Pathol. 2016;40(2):244-252.

8. Osunkoya AO. Mucinous and secondary tumors of the prostate. Modern Pathology. 2018;(31):S80-S95.

9. Samaratunga H, Delahunt B, Srigley JR, et al. Mucinous adenocarcinoma of prostate and prostatic adenocarcinoma with mucinous components: a clinicopathological analysis of 143 cases. Histopathology. 2017;71(4):641-647.
10. Osunkoya AO, Epstein JI. Primary mucin-producing urothelial-type adenocarcinoma of prostate: report of 15 cases. Am J Surg Pathol. 2007;31(9):1323-1329.

11. Osunkoya AO, Nielsen ME, Epstein JI. Prognosis of Mucinous Adenocarcinoma of the Prostate Treated by Radical Prostatectomy. Am J Surg Pathol. 2008;32(3):468-472.

12. Enciu M, Aschie M, Deacu M, et al. Morphological characteristics of a mucinous adenocarcinoma of the prostate: differential diagnosis considerations. Rom J Morphol Embryol. 2013;54(1):191-241.

13. McNeal JE, Alroy J, Villers A, et al. Mucinous differentiation in prostatic adenocarcinoma. Hum Pathol. 1991;22(10):979-988.

14. Westphalen AC, Fergus V, Kurhanewicz J, et al. Mucinous adenocarcinoma of the prostate: MRI and MR spectroscopy features. Am J Roentgenol. 2009;193(3):W238-W327.

15. Outwater E, Schiebler ML, Tomaszewski JE, et al. Mucinous carcinomas involving the prostate: atypical findings at MR imaging $J$ Magn Reson Imaging. 1992;2(5):597-600.

16. Pokorny MR, de Rooij M, Duncan E, et al. Prospective study of diagnostic accuracy comparing prostate cancer detection by transrectal ultrasound-guided biopsy versus magnetic resonance (MR) imaging with subsequent MR-guided biopsy in men without previous prostate biopsies. Eur Urol. 2014;66(1):22-29.

17. Ro JY, Grignon DJ, Ayala AG, et al. Mucinous adenocarcinoma of the prostate: histochemical and immunohistochemical studies. Hum Pathol. 1990;21(6):593-600.

18. Johnson H, Zhou M, Osunkoya AO. ERG expression in mucinous prostatic adenocarcinoma and prostatic adenocarcinoma with mucinous features: comparison with conventional prostatic adenocarcinoma. Hum Pathol. 2013;44(10):2241-2246.

19. Han B, Mehra R, Suleman K, et al. Characterization of ETS gene aberrations in select histologic variants of prostate carcinoma. Mod Pathol. 2009;22(9):1176-1185.

20. Osunkoya AO, Adsay NV, Cohen C, et al. MUC2 expression in primary mucinous and nonmucinous adenocarcinoma of the prostate: an analysis of 50 cases on radical prostatectomy. Mod Pathol. 2008;21(7):789-794. 